\title{
Hand Hygiene: Knowledge and Attitudes of Fourth-Year Clerkship Medical Students at Alfaisal University, College of Medicine, Riyadh, Saudi Arabia
}

\author{
Reem Hamadah ${ }^{1}$, Razan Kharraz ${ }^{1}$, Airabab Alshanqity ${ }^{1}$, Danah AlFawaz ${ }^{1}$, Abdulaziz M. \\ Eshaq $^{2}$, Ahmed Abu-Zaid ${ }^{3}$ \\ 1. College of Medicine, Alfaisal University 2. College of Medicine, Alfaisal University, Riyadh, USA 3. \\ College of Medicine, Alfaisal University, Riyadh, SAU
}

$\square$ Corresponding author: Ahmed Abu-Zaid, aabuzaid@live.com

Disclosures can be found in Additional Information at the end of the article

\section{Abstract}

Introduction: Little is known about the clerkship (clinical) medical students' knowledge of hand hygiene as the single most important precautionary measure to reduce nosocomial healthcareassociated infections. The aim of this study is to explore the knowledge of, and attitudes towards, hand hygiene practices among fourth-year clerkship medical students at Alfaisal University, College of Medicine, Riyadh, Saudi Arabia.

Materials and Methods: A cross-sectional, paper-based, Yes/No formatted questionnaire was administered to explore the students' knowledge of, and attitudes towards, hand hygiene practices. Data were decoded in Microsoft Excel sheet and presented as numbers and percentages.

Results: One hundred and eleven students ( $n=111 / 147)$ participated in the questionnaire (response rate: 76\%). Although the majority of students had a fair knowledge of hand hygiene practices, a number of them had some misconceptions. Only $14 \%$ of students correctly agreed to the statement: "Traditional hand washing (water, plus regular soap) decreases the number of germs." Furthermore, only 32\% of students correctly answered that "hand washing with a regular soap, instead of an antiseptic soap, is better in limiting the transmission of clostridium difficile infections". Almost all students (93\%) agreed to the importance of hand hygiene education in medical curricula and its awareness in healthcare centers. Despite the importance of hand hygiene, only $13 \%$ of students reviewed the respective WHO and CDC guidelines before starting their clinical training in the teaching hospital.

Received 08/01/2015

Review began 08/04/2015 Review ended 08/16/2015 Published 08/24/2015

\section{(C) Copyright 2015}

Hamadah et al. This is an open access article distributed under the terms of the Creative Commons Attribution License CC-BY 3.0., which permits unrestricted use, distribution, and reproduction in any medium, provided the original author and source are credited.
Discussion: The students' inadequate knowledge about hand hygiene needs to be enriched by well-structured curricular and extra-curricular programs as well as more positive attitudes by healthcare workers.

Categories: Medical Education, Public Health, Environmental Health

Keywords: hand hygiene, infection, healthcare, knowledge, attitude, clerkship, medical students, saudi arabia, medical education, curriculum

\section{Introduction}

Healthcare-associated infections (HAIs) bear huge burdens on healthcare systems. More

\section{How to cite this article}

Hamadah R, Kharraz R, Alshanqity A, et al. (August 24, 2015) Hand Hygiene: Knowledge and Attitudes of Fourth-Year Clerkship Medical Students at Alfaisal University, College of Medicine, Riyadh, Saudi Arabia. Cureus 7(8): e310. DOI 10.7759/cureus.310 
precisely, HAIs are associated with lengthy hospitalization, long-term disability, higher microbial drug resistance, increased morbidity, greater mortality, and extra healthcare-related costs [1]. Compliance of all healthcare workers (nurses, physicians, residents, and students) to the universally agreed standard infection control precautions is identified as an effective measure to control and prevent the occurrence of HAIs [2]. These measures not merely protect patients, but the healthcare workers, too [3].

Clerkship (clinical) medical students are key players in any healthcare teams and are greatly involved in the delivery of patient care. Moreover, during their clinical training, they rotate in infection-sensitive floors, such as: labor and delivery, intensive care units, neonatal intensive care units, and operating rooms, where greater requirements of sterility and infection control are highly demanded. Despite the significant impact of HAIs on the safety and cost of healthcare systems, priority consideration of HAIs education in pre-clerkship and clerkship medical curricula has yet to be reinforced [1]. As a result, largely due to lack of knowledge and skills, clerkship students entering clinical training are at a greater risk of causing HAIs to the patients.

Hand hygiene is regarded to be the single most central precautionary measure to prevent HAIs [4]. This notion is supported in the guidelines established by the World Health Organization (WHO) [5] and Centers for Disease Control and Prevention (CDC) [6].

Little is known about the clerkship students' knowledge of hand hygiene as one of the infection control measures. Exploring medical students' knowledge of, and attitudes towards, hand hygiene are of high importance to public health policy makers and medical educators. Such exploration is expected to identify the curricular needs and, therefore, can be appropriately incorporated into the pre-clerkship and clerkship medical curricula to equip students with satisfactory knowledge and skills. In the short- and long-term, such curricular incorporation is expected to decrease the rate of nosocomial HAIs that could be caused by clerkship medical students. Although there are a number of reports concerning the knowledge of hand hygiene among medical students in many countries, Saudi Arabia has greatly lagged behind in this aspect [7-8].

The aim of this study is to explore the knowledge of, and attitudes towards, hand hygiene among fourth-year clerkship medical students at Alfaisal University, College of Medicine, Riyadh, Saudi Arabia.

\section{Materials And Methods}

A cross-sectional study was conducted during the fall semester of 2014-2015. The participants were fourth-year clerkship medical students enrolled in Alfaisal University, College of Medicine who were entering clinical training at a single teaching hospital in Riyadh, Saudi Arabia. This study was approved by the Institutional Review Board (IRB) of Alfaisal University (approval \#2015-076).

Students were requested to complete a paper-based questionnaire. The questionnaire was administered during an academic lecture, and students were given 10 minutes to complete the questionnaire. The questionnaire was administered to explore students' demographical data as well as their knowledge of, and attitudes towards, hand hygiene.

Demographic data included gender and previous attendance of scientific meetings (conferences, symposia, seminars, workshops, etc.) about hand hygiene. Knowledge of hand hygiene was examined by 18 Yes/No formatted questions; they were scientifically based objective questions and explored information about gloves, antiseptic soaps, and sanitizers as 


\section{Cureus}

well as hand hygiene concepts and techniques. Attitudes towards hand hygiene were examined by five Yes/No formatted questions; they were opinion-based subjective questions and explored aspects relating to hand hygiene in terms of importance, education, compliance, and role models.

The questionnaire was developed based on a literature review (previous studies as well as WHO and CDC guidelines). All questions were peer-reviewed by two public health/infection control healthcare professionals and piloted on a group of students to ensure proper understanding and interpretation of questions.

After collecting the responses, data were decoded by using Microsoft Excel sheet (Microsoft Company, Redmond, WA, USA). Categorical data were presented as numbers and percentages.

\section{Results}

One hundred and eleven students ( $\mathrm{n}=111 / 147)$ participated in the questionnaire with an overall response rate of $76 \%$. Around $57 \%$ and $43 \%$ of students were males and females, respectively. Around $7 \%$ of students attended previous scientific meetings about hand hygiene.

Table 1 exhibits the students' knowledge of hand hygiene (expressed as percentages of correct answers).

\section{\begin{tabular}{l|l}
\hline \# & Question \\
\hline 1 & Traditional hand washing (water plus regular soap) decreases the number of germs \\
\hline
\end{tabular}}

2 Using gloves eliminates the necessity to wash hands

3 Hand washing is required before and after wearing gloves

4 Using instant hand sanitizer to quickly wash hands is always adequate

$5 \quad$ Must use anti-septic soap for proper hand washing

6 Must use both anti-septic soap plus hand sanitizer for proper hand washing

7 After washing hands, turn off water taps with your hands

8 After washing hands, turn off taps using piece of paper towel

9

Perform hand hygiene only before encountering patient (even without performing physical examination)

Perform hand hygiene only after encountering patient (even without performing physical

\section{Correct}

Answer

n (\%) Answer 15 


\section{Cureus}

10 examination)

Perform hand hygiene before and after encountering each patient (even without doing physical examination)

12 Enforce hand hygiene only before physically examining the patient

13 Enforce hand hygiene only after physically examining the patient

14 Enforce hand hygiene before and after physically examining the patient

15 Implement hand hygiene only after contact with secretions/bodily fluids (respiratory secretions, saliva, vomit and blood)

On unsoiled hands, an alcohol-based hand rub is recommended over an anti-septic soap hand washing

17 On unsoiled hands, an alcohol-based hand rub is recommended over a 3-minute surgical scrub

\section{TABLE 1: Students' knowledge of hand hygiene}

Table 2 shows the students' attitudes towards several aspects concerning hand hygiene.

\# Question

Before starting my clinical training, I reviewed the respective WHO and CDC guidelines for hand hygiene.

2 Healthcare providers are educating patients and their families about hand hygiene and its importance.

3 I lack proper hand hygiene practices because no living examples (that is, healthcare providers) are performing them.

4

Proper hand hygiene is an important matter to be emphasized in medical curricula and healthcare centers.

5 Improper hand hygiene contributes to a patient's morbidity and mortality.
Yes $\mathbf{n}$ No $\mathrm{n}$

(\%)

$14(13) \quad 97(87)$

$43(39) \quad 68(61)$

$67(60) \quad 44(40)$

\section{TABLE 2: Students' perceived attitudes towards hand hygiene}




\section{Discussion}

Studies from Saudi Arabia that explored clerkship medical students' knowledge of hand hygiene are limited. To the best of our knowledge, there are only two related studies conducted at Qassim University, College of Medicine, Qassim, Saudi Arabia [7] and King Faisal University,

College of Medicine, Dammam, Saudi Arabia [8]. Our current study represents the third study so far; it beneficially contributes to the production of data generalization about clerkship students' knowledge of hand hygiene in Saudi Arabia. The remaining 10 Saudi Arabian medical colleges are encouraged to follow in our footsteps in conducting similar studies - this is a knowledge deficit and stimulating field for further research.

Our study endeavored to examine clerkship medical students' knowledge of, and attitudes towards, hand hygiene as one of the most important infection control measures. It revealed that students' knowledge of hand hygiene is inadequate. Several students wrongly answered some basic hand hygiene questions. Our results were largely similar to other studies conducted elsewhere in Saudi Arabia [7-8], India [9], China [1], Namibia [10], United Kingdom [11], and Brazil [2].

According to CDC guidelines on hand hygiene, washing hands with water and regular soap is the best available method to decrease the number of microbes on them in the vast majority of circumstances [12]. In our study, only 15 students (14\%) correctly answered that "Traditional hand washing (water plus regular soap) decreases the number of germs". Conversely, in an Indian study, 88\% of clerkship medical students answered this question correctly [9].

When water and soap are unavailable - which can be the case in several occasions in healthcare settings - students should look for an alternative, such as hand sanitizers. Students exhibited several major misconceptions regarding the use of hand sanitizers. This can generally be attributed to the students' lack of knowledge. For example, 69 students (62\%) falsely agreed that "Using instant hand sanitizer to quickly wash hands is adequate". Students should recognize the difference between alcohol-based and non-alcohol-based hand sanitizers. For effective killing/elimination of germs, several studies have shown that highly-concentrated alcohol-based hand sanitizers (60-95\% alcohol concentration) are more superior to lowconcentrated alcohol-based (less than 60\% alcohol concentration) and non-alcohol-based hand sanitizers [13-14]. The disadvantages of non-alcohol-based hand sanitizers, such as skin irritation and tendency to develop resistance to the sanitizing agents, should be enlightened [13-14]. Moreover, students should recognize that alcohol-based hand sanitizers can eliminate several kinds of microbes very effectively - but only when used properly [15]. Such improper use of common hand sanitizers that preclude their antimicrobial effectiveness include an inadequate amount or rapid wiping off of sanitizer before hands have completely dried [16].

In addition, students should be aware that traditional hand washing (water, plus regular soap) has been demonstrated to be more effective at inactivating and eliminating particular kinds of germs, such as clostridium difficile-associated infections in suspected individuals/patients [17]. This is a piece of information that can be easily missed by physicians, nurses, and medical students; only 36 students (32\%) answered it correctly. Likewise, in a Chinese study conducted by Huang, et al., only $23.5 \%$ of the students responded correctly [1].

Besides, students should understand that "on unsoiled hands, an alcohol-based hand rub is recommended over an antiseptic soap for proper hand hygiene", as it is quicker and better endured [14]. Only 42 students (38\%) agreed correctly to this fact. Students should also recognize that hand sanitizers are infective and not recommended when hands are greatly contaminated, soiled, or greasy [14-15]. Instead, hand washing with water and regular/antiseptic soap is preferred in such conditions and remains the best modality for the 
control of infection spread, especially when they are visibly dirty and unclean [12].

Up to this moment, there are no well-documented and established recommendations for using both hand sanitizers and antiseptic soap to clean hands and achieve hand hygiene. Only 48 students (43\%) answered this question correctly.

An infection control preventive measure that is worthy of mentioning is the use of medical gloves [2]. According to the respective WHO guidelines, 1) the use of gloves does not eliminate the necessity to wash hands and 2) hand hygiene is required before and after wearing gloves [18]. In our study, students largely showed satisfactory understanding of the two abovementioned WHO guidelines (percentage of students' correct answers were $88 \%$ and $72 \%$, respectively).

Students showed appropriate awareness of hand hygiene in terms of indications and techniques. Hand hygiene should be performed before and after each patient encounter (regardless of performing physical examination) [19], and 82 students (74\%) answered this question correctly. Moreover, 81 students (73\%) correctly answered that hand hygiene should be enforced before and after each physical examination. In addition, while 86 students (77\%) properly disagreed to the following statement: "After washing hands, turn off water taps with your hands" [18], only 71 students (64\%) properly agreed to the following statement: "After washing hands, turn off water tap using piece of paper towel" [20].

It should be noted that the relatively appropriate awareness of hand hygiene in the context of 1) patient encounter, 2) physical examination, and 3) hand washing techniques can be attributed to the intensive pre-clerkship training the students have received in the "Clinical Skills" courses at our college.

In our study, students showed positive attitudes towards hand hygiene. More than $90 \%$ of the students agreed that "Proper hand hygiene is an important matter to be emphasized in medical curricula and healthcare centers" and that "Improper hand hygiene significantly contributes to a patient's morbidity and mortality". It is of high significance to spread the awareness about hygiene among undergraduate clerkship medical students as this will be reflected on their behaviors later on when they become professional healthcare providers. Obviously, the higher the level of education/awareness about hand hygiene standards, the more precautions will be taken, and the less ensued nosocomial HAI rates [21]. Despite the students' realization of the significance of hand hygiene, only 14 students (13\%) stated that they have reviewed the CDC and WHO guidelines for hand hygiene prior to starting their clerkship clinical training.

Sixty-seven students (60\%) declared that one of the reasons accounting for their lack of proper hand hygiene is related to absence of living examples (that is, healthcare providers: physicians, residents, nurses, interns) adhering to the hand hygiene guidelines. Previous studies showed that students' hand hygiene behaviors are substantially influenced by their role models' (i.e., physicians) behaviors towards hand hygiene [22-23]. More specifically, in a Canadian study by Janq, et al., it was revealed that medical students' hand hygiene were significantly affected by the hand hygiene exercised by their physicians; there was a negative impact on students' hand hygiene as a result of non-compliance to proper hand hygiene by their senior physicians [24]. Physician role models and teamwork efforts are two valuable means to modulate and encourage proper hand hygiene by students.

Only 43 students (39\%) asserted that they were "educating patients and their families about hand hygiene and its importance". This is a worrisome issue. Clerkship medical students' roles should evolve from merely "learners" to include "public health advocators", "patient safety ambassadors", and "valuable mentors/roles models" by promoting hand hygiene compliance 
among patients and healthcare workers, too. These qualities should be cultivated in medical students early in pre-clerkship curricula and strongly reinforced in clerkship clinical training by their teaching physicians.

The inadequate knowledge of our students can be attributed to two main factors. First, in our pre-clerkship curriculum (as it is the case in most medical curricula in developed [11] and developing [24] countries), standard precautions, infection control measures, and hand hygiene are not sufficiently taught as independent courses or incorporated into other core courses, such as microbiology and clinical skills. Moreover, formal comprehensive reinforcing/teaching of such infection control fundamentals does not exist in the clerkship curricula either. Second, programs and campaigns, which raise awareness about hand hygiene, are largely lacking in oncampus and healthcare center settings.

There are some steps that can be taken to enhance the hand hygiene awareness among clerkship medical students. First, the formal integration of a comprehensive public health curriculum in pre-clinical (pre-clerkship) and clinical (clerkship) years is needed [25]. The significance of hand hygiene as one of the most effective infection control measures in the undergraduate curriculum should be highlighted through formal teaching and assessment, especially in clinical settings. Such a highlighting initiative is anticipated to 1) enhance students' knowledge and 2) aid in promoting a positive culture towards hand hygiene [9]. For example, in a study conducted in Kenya, there was a remarkable advancement in knowledge about hand hygiene among school students after implementing a program about hygiene in their curriculum [26]. Nonetheless, previous studies showed that teaching infection control to medical students is challenging in terms of developing appropriate curricula and cultivating proper attitudes towards infection control [27].

Second, using the systemic Hand Hygiene Self-Assessment Framework tool can help in generating an analysis of hand hygiene of all healthcare workers at a particular healthcare center [28]. The tool works by giving information about current resources and achievements as well as identifying the improper hand hygiene that needs to be looked at and resolved. Third, organizing promotional campaigns and spreading awareness in a creative way [29] by demonstrating the importance of hand hygiene and its emphasis in the contribution to a patient's morbidity and mortality would thus encourage its proper practice. Fourth, providing continuous feedback from mentors is useful in reinforcing the importance of hand hygiene [30].

There are a few limitations to our study. First, the study design was cross-sectional; differing results could have been obtained if another time-frame had been chosen. Second, all questions were Yes/No formatted questions, and accordingly, answers were liable to random correct guessing (without penalty for wrong answers). Third, the use of opposing statements in subsequent questions of the questionnaire may have forced respondents more towards the guessing mode for answering.

\section{Conclusions}

Clerkship medical students had some misconceptions about hand hygiene that reflected their inadequate knowledge. Medical educators should design well-structured pre-clerkship and clerkship curricula that guarantee satisfactory knowledge of infection control measures and hand hygiene. Public awareness and creative campaigns about hand hygiene are also encouraged. Developing positive attitudes towards hand hygiene and the students' compliance to such should be underscored as being essential to clinical practice and reducing the rate of HAIs. Incorporating formal curricular assessments is one plausible method to cultivate this compliance. Students' adequate knowledge of hand hygiene is expected to decrease the rate of nosocomial HAIs that could be incurred unintentionally or negligibly by the clerkship medical students. Future research projects include exploring students' perception on barriers towards 
hand hygiene education and practice as well as exploring the impact of mentoring role models (physicians, nurses, etc.) towards proper hand hygiene.

\section{Additional Information \\ Disclosures}

Human subjects: Consent was obtained by all participants in this study. Institutional Review Board (IRB) at Alfaisal University issued approval 2015-076. Animal subjects: All authors have confirmed that this study did not involve animal subjects or tissue. Conflicts of interest: In compliance with the ICMJE uniform disclosure form, all authors declare the following:

Payment/services info: All authors have declared that no financial support was received from any organization for the submitted work. Financial relationships: All authors have declared that they have no financial relationships at present or within the previous three years with any organizations that might have an interest in the submitted work. Other relationships: All authors have declared that there are no other relationships or activities that could appear to have influenced the submitted work.

\section{References}

1. Huang Y, Xie W, Zeng J, Law F, Ba-Thein W: Limited knowledge and practice of Chinese medical students regarding health-care associated infections. J Infect Dev Ctries. 2013, 7:144151. 10.3855/jidc.3099

2. García-Zapata MRC, Silva e Souza AC, Guimarães JV, Tipple AFV, Prado MA, García-Zapata MTA: Standard precautions: knowledge and practice among nursing and medical students in a teaching hospital in Brazil. Int J Infect Control. 2010, 6:122-123. 10.3396/ijic.V6i1.005.10

3. Garner JS: Guideline for isolation precautions in hospitals. Hospital Infection Control Practices Advisory Committee. Infect Control Hosp Epidemiol. 1996, 17:53-80.

4. Graf K, Chaberny IF, Vonberg RP: Beliefs about hand hygiene: a survey in medical students in their first clinical year. Am J Infect Control. 2011, 39:885-888. 10.1016/j.ajic.2010.08.025

5. WHO. WHO Guidelines on Hand Hygiene in Health Care: a SummaryWHO . (2009). Accessed: November 22, 2014: http://www.who.int/gpsc/5may/tools/who_guidelineshandhygiene_summary.pdf.

6. Boyce JM, Pittet D; Healthcare Infection Control Practices Advisory Committee; HICPAC/SHEA/APIC/IDSA Hand Hygiene Task Force: Guideline for Hand Hygiene in HealthCare Settings. Recommendations of the Healthcare Infection Control Practices Advisory Committee and the HIPAC/SHEA/APIC/IDSA Hand Hygiene Task Force. Am J Infect Control. 2002, 30:S1-S46.

7. Al Kadi A, Salati SA: Hand Hygiene Practices among Medical Students. Interdiscip Perspect Infect Dis. 2012, 2012:679129. 10.1155/2012/679129

8. Amin TT, Al Noaim KI, Bu Saad MA, Al Malhm TA, Al Mulhim AA, Al Awas MA: Standard precautions and infection control, medical students' knowledge and behavior at a Saudi university: the need for change. Glob J Health Sci. 2013, 21:114-125. 0.5539/gjhs.v5n4p114

9. Ayub A, Goyal A, Kotwal A, Kulkarni A, Kotwal A, Mahen A: Infection control practices in health care: Teaching and learning requirements of medical undergraduates. Med J Armed Forces India. 2013, 69:107-112. 10.1016/j.mjafi.2012.07.021

10. Ojulong J, Mitonga KH, Iipinge SN: Knowledge and attitudes of infection prevention and control among health sciences students at University of Namibia. Afr Health Sci. 2013, 13:1071-1078. 10.4314/ahs.v13i4.30

11. Mann CM, Wood A: How much do medical students know about infection control? . J Hosp Infect. 2006, 64:366-367. 10.1016/j.jhin.2006.06.030

12. CDC. Handwashing. Show Me the Science - When to Use Hand Sanitizer . (2015). Accessed: Jul 25, 2014: http://www.cdc.gov/handwashing/show-me-the-science-hand-sanitizer.html? mobile=nocontent\#s7-ten.

13. Kampf G, Kramer A: Epidemiologic background of hand hygiene and evaluation of the most important agents for scrubs and rubs. Clin Microbiol Rev. 2004, 17:863-893.

10.1128/CMR.17.4.863-893.2004 
14. Todd EC, Michaels BS, Holah J, Smith D, Greig JD, Bartleson CA: Outbreaks where food workers have been implicated in the spread of foodborne disease. Part 10. Alcohol-based antiseptics for hand disinfection and a comparison of their effectiveness with soaps. J Food Pro. 2010, 73:2128-2140.

15. Edmonds SL, Mann J, McCormack RR, Macinga DR, Fricker CM, Arbogast JW, Dolan MJ: SaniTwice: a novel approach to hand hygiene for reducing bacterial contamination on hands when soap and water are unavailable. J Food Prot. 2010, 73:2296-2300.

16. Kampf G, Marschall S, Eggerstedt S, Ostermeyer C: Efficacy of ethanol-based hand foams using clinically relevant amounts: a cross-over controlled study among healthy volunteers. BMC Infect Dis. 2010, 10:78. 10.1186/1471-2334-10-78

17. Oughton MT, Loo VG, Dendukuri N, Fenn S, Libman MD: Hand hygiene with soap and water is superior to alcohol rub and antiseptic wipes for removal of Clostridium difficile. Infect Control Hosp Epidemiol. 2009, 30:939-944. 10.1086/605322

18. WHO. Hand Hygiene: Why, How \& When?. (2009). Accessed: November 22, 2014: http://www.who.int/gpsc/5may/Hand_Hygiene_Why_How_and_When_Brochure.pdf.

19. CDC. Dialysis Safety: Protocol for Hand Hygiene and Glove Use Observations . (2013). Accessed: November 22, 2014: http://www.cdc.gov/dialysis/prevention-tools/Protocol-handhygiene-glove-observations.html.

20. myVMC. Hand hygiene technique: hand washing with soap and water. Accessed: 22 November, 2014. Available from. (2013). Accessed: November 22, 2014:

http://www.myvmc.com/lifestyles/hand-hygiene-technique-hand-washing-with-soap-andwater/.

21. Herbert VG, Schlumm P, Kessler HH, Frings A: Knowledge of and Adherence to Hygiene Guidelines among Medical Students in Austria. Interdiscip Perspect Infect Dis. 2013, 2013:802930. 10.1155/2013/802930

22. van de Mortel TF, Apostolopoulou E, Petrikkos: A comparison of the hand hygiene knowledge, beliefs, and practices of Greek nursing and medical students. J Adv Nurs. 2010, 68:75-77. 10.1111/j.1365-2648.2011.05758.x

23. Jang JH, Wu S, Kirzner D, Moore C, Youssef G, Tong A, Lourenco J, Stewart RB, McCreight LJ, Green K, McGeer A: Focus group study of hand hygiene practice among healthcare workers in a teaching hospital in Toronto, Canada. Infect Control Hosp Epidemiol. 2010, 21:144-150. 10.1086/649792

24. Anwar MA, Rabbi S, Masroor M, Majeed F, Andrades M, Baqi S: Self-reported practices of hand hygiene among the trainees of a teaching hospital in a resource limited country. J Pak Med Assoc. 2009, 59:631-634.

25. Basu S, Roberts C: Towards a public health curriculum in undergraduate medicine . Educ Health (Abingdon). 2012, 25:98-104. 10.4103/1357-6283.103456

26. Patel MK, Harris JR, Juliao P, Nygren B, Were V, Kola S, Sadumah I, Faith SH, Otieno R, Obure A, Hoekstra RM, Quick R: Impact of a hygiene curriculum and the installation of simple handwashing and drinking water stations in rural Kenyan primary schools on student health and hygiene practices. Am J Trop Med Hyg. 2012, 87:594-601. 10.4269/ajtmh.2012.11-0494

27. Hunt DC, Mohammudally A, Stone SP, Dacre J: Hand-hygiene behaviour, attitudes and beliefs in first year clinical medical students. J Hosp Infect. 2005, 59:371-373.

10.1016/j.jhin.2004.09.002

28. WHO. Hand Hygiene Self-Assessment Framework 2010. (2010). Accessed: November 22, 2014: http://www.who.int/gpsc/country_work/hhsa_framework_October_2010.pdf.

29. CDC. Hand Hygiene in Healthcare Settings: Promotional Campaigns . (2015). Accessed: April 10, 2015: http://www.cdc.gov/handhygiene/resources.html.

30. Longtin Y, Sax H, Allegranzi B, Schneider F: Pittet D:s Videos in clinical medicine. Hand hygiene. N Engl J Med. 2011, 364:e24. 10.1056/NEJMvcm0903599 